\title{
The host of the Type I SLSN 2017egm
}

\section{A young, sub-solar metallicity environment in a massive spiral galaxy}

\author{
L. Izzo ${ }^{1}$, C. C. Thöne ${ }^{1}$, R. García-Benito ${ }^{1}$, A. de Ugarte Postigo ${ }^{1,2}$, Z. Cano ${ }^{1}$, D. A. Kann ${ }^{1}$, K. Bensch ${ }^{1}$, \\ M. Della Valle ${ }^{3,4}$, D. Galadí-Enríquez ${ }^{5}$, and R. P. Hedrosa ${ }^{5}$ \\ ${ }^{1}$ Instituto de Astrofisica de Andalucia (IAA-CSIC), Glorieta de la Astronomia s/n, 18008 Granada, Spain \\ e-mail: luca.izzo@gmail.com \\ 2 Dark Cosmology Centre, University of Copenhagen, Juliane Maries Vej 30, 2100 Copenhagen Ø, Denmark \\ 3 INAF - Capodimonte Observatory, Salita Moiariello 16, 80131 Napoli, Italy \\ 4 International Center for Relativistic Astrophysics, Piazzale della Repubblica, 2, 65122 Pescara, Italy \\ 5 Centro Astronómico Hispano-Alemán, Calar Alto, CSIC-MPG, Sierra de los Filabres-04550 Gérgal (Almería), Spain
}

Received 12 August 2017 / Accepted 1 October 2017

\begin{abstract}
Context. Type I superluminous supernova (SLSN) host galaxies are predominantly low-metallicity, highly star-forming (SF) dwarfs One of the current key questions is whether Type I SLSNe can only occur in such environments and hosts.

Aims. Here we present an integral-field study of the massive, high-metallicity spiral NGC 3191, the host of SN 2017egm, the closest Type I SLSN known to date. We use data from PMAS/CAHA and the public MaNGA survey to shed light on the properties of the SLSN site and the origin of star formation in this non-starburst spiral galaxy.

Methods. We map the physical properties of different H II regions throughout the galaxy and characterise their stellar populations using the STARLIGHT fitting code. Kinematical information allows us to study a possible interaction with its neighbouring galaxy as the origin of recent star formation activity which could have caused the SLSN.

Results. NGC 3191 shows intense star formation in the western part with three large SF regions of low metallicity. Taking only the properties of emitting gas, the central regions of the host have a higher metallicity, a lower specific star formation rate, and lower ionisation. Modelling the stellar populations gives a different picture: the SLSN region has two dominant stellar populations with different ages, the younger one with an age of 2-10 Myr and lower metallicity, likely the population from which the SN progenitor originated. Emission line kinematics of NGC 3191 show indications of interaction with its neighbour MCG+08-19-017 at $\sim 45 \mathrm{kpc}$, which might be responsible for the recent starburst. In fact, this galaxy pair has hosted a total of four SNe, 1988B (Type Ia), SN 2003ds (Type Ic in MCG+08-19-017), PTF10bgl (Type II), and 2017egm, underlying the enhanced SF in both galaxies due to interaction. Conclusions. Our study shows that care should be taken when interpreting global host and even gas properties without looking at the stellar population history of the region. The SLSNe seem to be consistent with massive stars $\left(>20 M_{\odot}\right)$ requiring low metallicity $\left(<0.6 Z_{\odot}\right)$, environments that can also occur in massive late-type galaxies, but not necessarily with starbursts.
\end{abstract}

Key words. supernovae: individual: 2017egm - galaxies: general

\section{Introduction}

Superluminous supernovae (SLSNe) are defined as supernovae $(\mathrm{SNe})$ with absolute peak magnitudes in any optical band of $<-21 \mathrm{mag}$ (Gal-Yam 2012) and were detected for the first time a decade ago, thanks to the advent of new untargeted large-scale surveys probing all types of galaxies (Kaiser \& Pan-STARRS Team 2005; Drake et al. 2009; Law et al. 2009; Tonry et al. 2012). SLSNe have been divided into three main classes: 1) Type I SLSNe or H-poor SNe characterised by the absence of hydrogen in the spectra (Quimby et al. 2007, 2011; Pastorello et al. 2010; Chomiuk et al. 2011); 2) Type II SLSNe or H-rich that show narrow hydrogen lines, likely due to a massive surrounding $\mathrm{H}$-envelope, therefore basically making them high-luminosity Type IIn SNe (Ofek et al. 2007; Smith et al. 2007); and possibly 3) Type "R" SLSNe, which are SLSNe powered by radioactive decay of large amounts of Ni. Their high luminosities imply that their origins are different from those of normal $\mathrm{SNe}$, and the study of the immediate environment of these events can provide important information on physical properties of their progenitors which so far have remained undetected.

Indeed, several studies of SLSN host galaxy samples have revealed significant differences in the properties of Type I and Type II hosts (Leloudas et al. 2015; Perley et al. 2016; Lunnan et al. 2014; Schulze et al. 2016). Type I hosts are usually star-forming (SF) dwarf galaxies with low metallicities at the extreme end of the distribution in the BaldwinPhilips-Terlevich (BPT; Baldwin et al. 1981) diagram and likely even more extreme than gamma-ray burst (GRB) hosts (see e.g. Leloudas et al. 2015; Schulze et al. 2016; but see also Lunnan et al. 2014). In addition, they have been found in very young stellar populations, indicating that Type I SLSNe might be the first and most massive stars to end in a SN explosion after the onset of a new starburst episode in their galaxy or HII region (Thöne et al. 2015). Type II hosts seem to be more metal rich and within the bulk of emission-line galaxies in the SDSS. At higher redshifts, Type I SLSN hosts evolve in line with the most star-forming galaxies towards higher-mass galaxies with higher absolute star formation rates (SFRs), but still seem to show a 

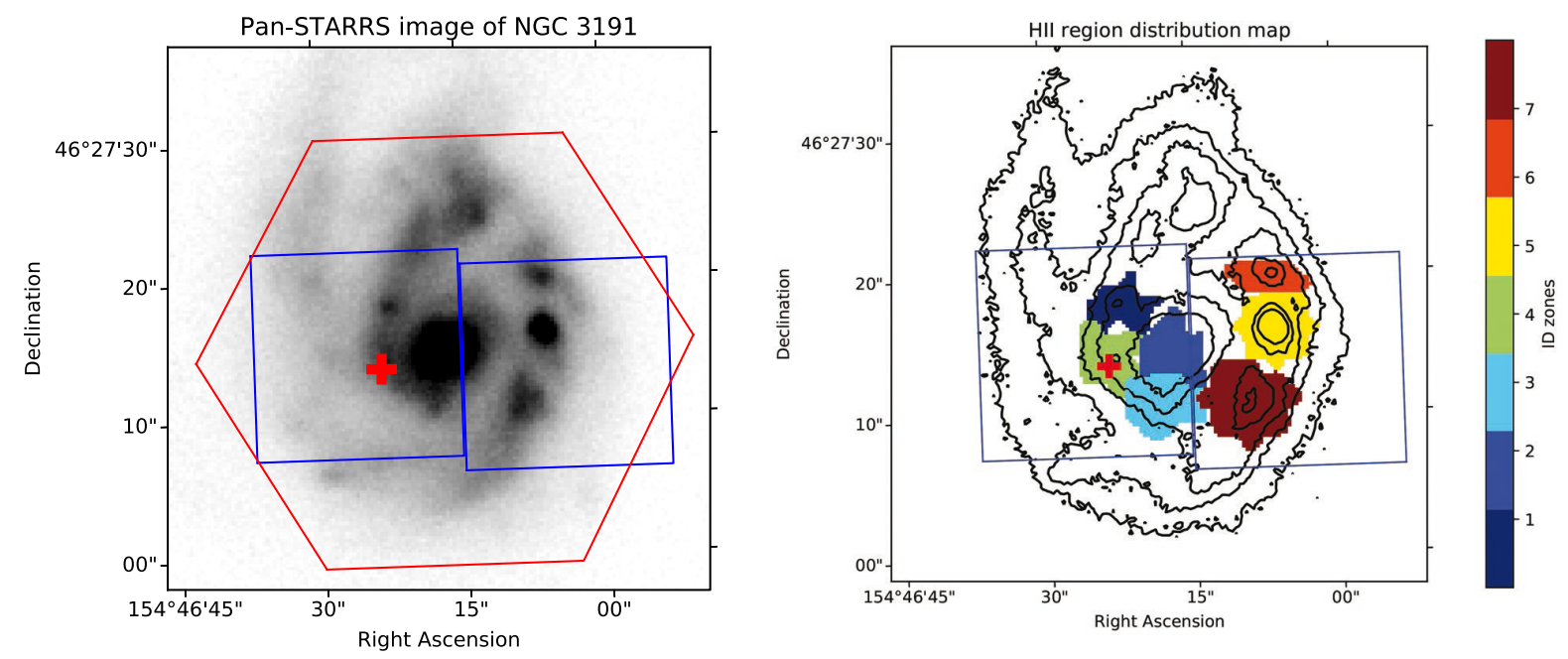

Fig. 1. Left panel: Pan-STARRS $r^{\prime}$-band image of NGC 3191 with the PMAS (blue squares) and the MaNGA fields of view (red hexagon) superposed. Right panel: map of the H II regions obtained with the code described in the text and applied to the PMAS cubes. The SLSN 2017egm position is marked with a red cross in both panels. The levels of the isophotes, from the outermost to the innermost, are 21.86, 21.11, 20.66, 20.22, and $19.91 \mathrm{mag} / \mathrm{arcsec}$.

metallicity cut-off at $\sim 0.4$ solar and a tendency towards lowmass hosts (Schulze et al. 2016).

In this paper, we present integral field unit (IFU) spectroscopic data of NGC 3191, the host galaxy of SN 2017egm, a Type I SLSN discovered by the Gaia satellite (Delgado et al. 2017) on May 23, 2017. Detailed analysis of its early optical spectra revealed its Type I SLSN nature (Dong et al. 2017; Nicholl et al. 2017). The SLSN reached its optical maximum on June 20, 2017, with an absolute magnitude of $M_{\mathrm{U}} \sim-22$ mag. As pointed out in Nicholl et al. (2017), Bose et al. (2017), and Chen et al. (2017b), the host seems to be the first Type I host with super-solar metallicity. In this work, we analyse the properties of different regions in the host galaxy, and in the SLSN location, providing a more detailed analysis of the metallicity and the star formation rate distributions around the SLSN environment and several other $\mathrm{H}$ II regions. Resolved spectral modelling across the host galaxy gives us important clues to the different underlying stellar populations, their ages, and their metallicities, allowing us a more detailed insight into this apparently very peculiar SLSN-I environment.

\section{Observations}

We observed the host galaxy of SN 2017egm with the Potsdam Multi-Aperture Spectrophotometer (PMAS) mounted on the $3.5 \mathrm{~m}$ telescope of the Calar Alto Observatory (Spain; Roth et al. 2005) on June 21, 2017. PMAS is an integral field spectrograph composed of $16 \times 16$ square elements in the lens array. We used the 1".0 spatial resolution configuration, which provides a field of view of $16^{\prime \prime} \times 16^{\prime \prime}$; the R600 grism was positioned at the grating position 146.5, which results in the wavelength range 4700-7700 $\AA$ with a spectral resolution of $1.55 \AA$ (resolving power $R \sim 600$ ) and the maximum efficiency centred around the rest-frame $\mathrm{H} \alpha$ region $(\sim 6600 \AA)$. The average seeing during the observations was of $1^{\prime \prime} .2$. The data were reduced with the P3D data reduction tools (Sandin et al. 2010) to obtain a final fluxcalibrated cube for each single observation.

Due to the dimensions of the host galaxy NGC 3191 (46" $\times$ $\left.37^{\prime \prime}\right)$ we were not able to cover the entire galaxy within a single PMAS shot. Given the limited time available, we decided to observe the SLSN region and the adjacent western half of the galaxy characterised by the presence of active $\mathrm{H}$ II regions, with two single exposures of $600 \mathrm{~s}$ each. The galaxy and the two regions covered by our PMAS observations are shown in the left panel of Fig. 1.

We complement our data set with the publicly available observations of NGC 3191 from the Mapping Nearby Galaxies at APO (MaNGA) survey (Law et al. 2015), available in DR14 of the $\operatorname{SDSS}^{1}$. The host galaxy, of which about $2 / 3$ are covered by the MaNGA data, was observed in January 2016, well before the appearance of the SLSN. Given the lower spatial resolution of the MaNGA survey (on average 2".5) we used the PMAS data for the identification and extraction of the H II regions in the host. For properties that need a higher spectral resolution (1.0 $\AA, R \sim 2000$ ), such as the estimate of the velocity distribution and stellar population properties, we used the MaNGA data. To ensure extraction of similar regions in the MaNGA as in the PMAS data we projected the World Coordinate System (WCS) of the Pan-STARRS image on the data cubes of both PMAS and MaNGA using the astropy python package (Astropy Collaboration et al. 2013), whose set of libraries has been extensively used in this work.

Finally, we use three public spectra of NGC 3191 and its neighbour galaxy MCG+08-19-017. The first spectrum covers the centre of NGC 3191 and was obtained in the context of the BOSS survey (SDSS-III, Dawson et al. 2013), with 2" fibres and an average resolving power of $R \sim 2000$ at $\sim 6000 \AA$. The other two spectra cover the brightest SF region in the west of NGC 3191 and the centre of MCG+08-19-017 and were obtained within the Legacy Survey (SDSS-I/II, York et al. 2000), with $3^{\prime \prime}$ fibres and an average resolving power of $R \sim 1800$.

\section{Emission line analysis}

The host galaxy of SN 2017egm is a spiral galaxy (type Sbc) at a redshift of $z=0.03072$ (Hakobyan et al. 2012) with an absolute magnitude of $M_{r}=-21.9$ (Taddia et al. 2016) and a

\footnotetext{
1 http://skyserver. sdss.org/dr14/en/tools/explore/
} Summary . aspx?id=1237658613587181631 


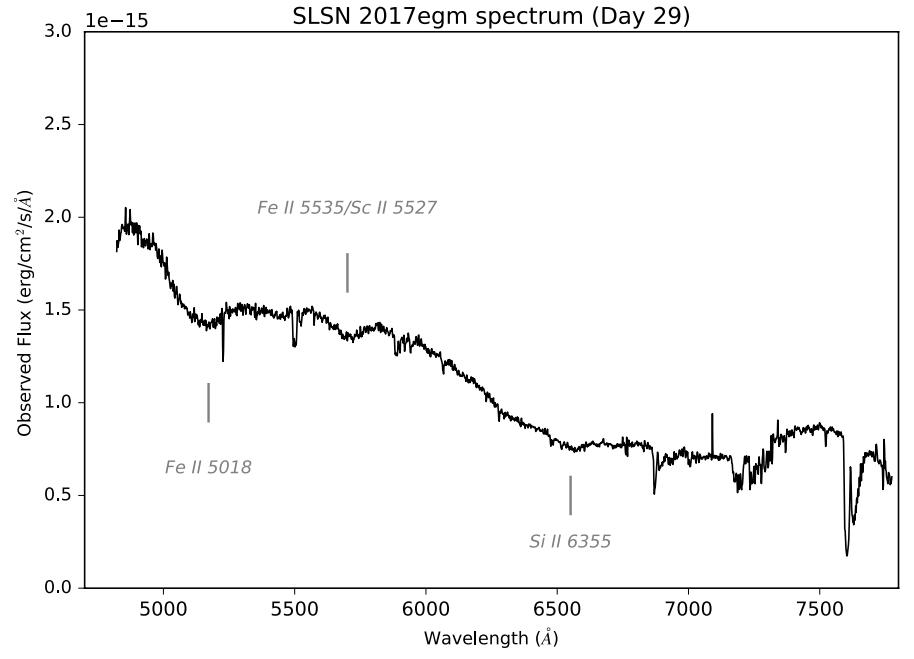

Fig. 2. Observed spectrum of SLSN 2017 egm 29 days after discovery, which we use as template to correct the PMAS data of region H II-4. We identified some typical features of Type I SLSNe such as Fe II $5018 \lambda$, the blend Fe II 5535 $\lambda$ - Sc II 5527 $\lambda$, and Si II 6355 $\lambda$.

Table 1. Coordinates (J2000.0) of the emission peaks for each H II region that we identified.

\begin{tabular}{lcc}
\hline \hline Region & RA & Dec \\
\hline H II-1 & $10^{\mathrm{h}} 19^{\mathrm{m}} 07.90^{\mathrm{s}}$ & $+46^{\mathrm{d}} 26^{\mathrm{m}} 48.35^{\mathrm{s}}$ \\
H II-2 & $10^{\mathrm{h}} 19^{\mathrm{m}} 07.81^{\mathrm{s}}$ & $+46^{\mathrm{d}} 26^{\mathrm{m}} 47.32^{\mathrm{s}}$ \\
H II-3 & $10^{\mathrm{h}} 19^{\mathrm{m}} 07.84^{\mathrm{s}}$ & $+46^{\mathrm{d}} 26^{\mathrm{m}} 46.58^{\mathrm{s}}$ \\
H II-4 & $10^{\mathrm{h}} 19^{\mathrm{m}} 07.93^{\mathrm{s}}$ & $+46^{\mathrm{d}} 26^{\mathrm{m}} 47.36^{\mathrm{s}}$ \\
H II-5 & $10^{\mathrm{h}} 19^{\mathrm{m}} 08.00^{\mathrm{s}}$ & $+46^{\mathrm{d}} 26^{\mathrm{m}} 48.14^{\mathrm{s}}$ \\
H II-6 & $10^{\mathrm{h}} 19^{\mathrm{m}} 08.00^{\mathrm{s}}$ & $+46^{\mathrm{d}} 26^{\mathrm{m}} 49.14^{\mathrm{s}}$ \\
H II-7 & $10^{\mathrm{h}} 19^{\mathrm{m}} 08.05^{\mathrm{s}}$ & $+46^{\mathrm{d}} 26^{\mathrm{m}} 46.91^{\mathrm{s}}$ \\
\hline
\end{tabular}

Notes. The H II-2 region corresponds to the galaxy centre, while H II-4 is the SLSN site.

stellar mass of $M_{*}=10^{10.61} M_{\odot}$ (Stoll et al. 2013). The PanSTARRS archival $r^{\prime}$-band image reveals the presence of several bright knots corresponding to highly star-forming $\mathrm{H}$ II regions in the galaxy. From the PMAS cubes we create a map of the $\mathrm{H} \alpha$ distribution by summing the flux of 15 pixels in wavelength centred at the corresponding redshifted emission line. The continuum is subtracted by taking the average flux of 10 pixels redwards and bluewards with respect to the $\mathrm{H} \alpha$ line and free of sky emission or telluric absorption lines.

In the $\mathrm{H} \alpha$ map we identify seven $\mathrm{H}$ II regions using a code derived from the HII-explorer package ${ }^{2}$ taking an $\mathrm{H} \alpha$ threshold for the peak flux for each region of $P=3 \times 10^{-16} \mathrm{erg} / \mathrm{cm}^{2} / \mathrm{s}$, corresponding to an intrinsic luminosity of $5 \times 10^{38} \mathrm{erg} / \mathrm{s}$ (as was done in Izzo et al. 2017). The identified H II regions are shown in the right panel of Fig. 1; their central coordinates are listed in Table 1 . H II regions 2 and 4 correspond to the galaxy centre and the SLSN position, respectively. Region 4 indeed shows spectral features from the actual SLSN, which at the time of our observations was close to its maximum luminosity (see Fig. 2). We also note that the H II-6 region has some defects in the MaNGA data which affect the extraction of an integrated spectrum of this region.

To correct the PMAS data for the underlying SN emission, we extracted the SLSN spectrum from the PMAS data using

\footnotetext{
2 For further details see http://lucagrb.weebly.com/hii.html
}

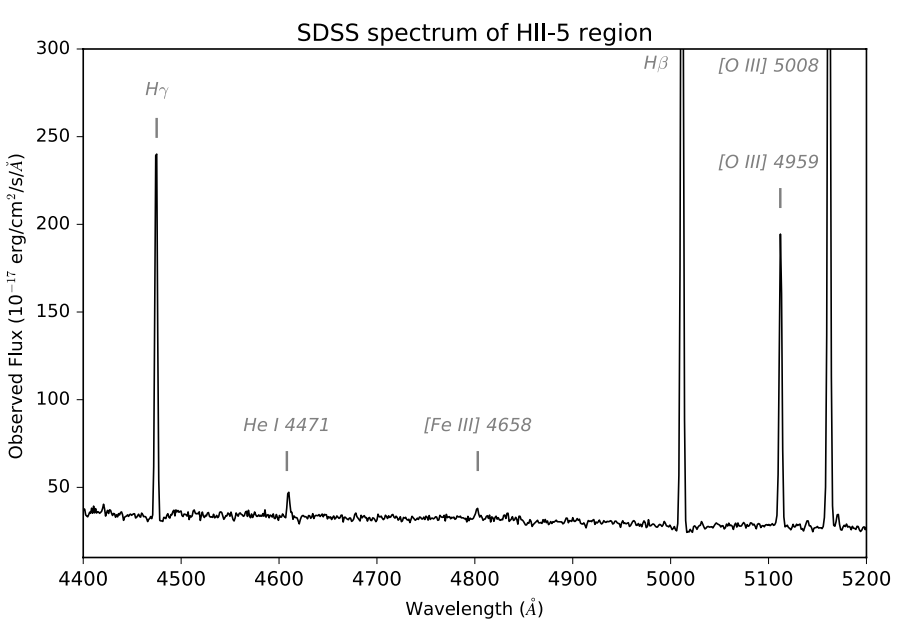

Fig. 3. Section of the SDSS spectrum of region HII-5, showing $\mathrm{He}$ I $4471 \lambda$ and a faint absorption trough at the bottom of the bright $\mathrm{H} \beta$ line.

the following process. We interpolated the MaNGA data with a power-law function to fit the continuum of the underlying H II region. Then we subtracted this model from the PMAS H II-4 region, after a pass by the ZAP software for the subtraction of sky and telluric emission lines (Soto et al. 2016). The final SN spectrum is shown in Fig. 2. Finally, in order to accurately measure the line fluxes, the SN spectrum was subtracted from the $\mathrm{H}$ II-4 region.

We do not find any absorption feature caused by an underlying stellar population around the $\mathrm{H} \beta$ line in the PMAS data cube, even in the spectra with the highest $\mathrm{S} / \mathrm{N}$ value (H II-5, 6, 7 regions). This is mainly caused by the low spectral resolution and by the low signal-to-noise ratio of some of the spectra. A spectrum of the region H II-5 can also be found in the SDSS-DR12 Science Archive Server ${ }^{3}$. In this spectrum we note the presence of a broad absorption feature at the $\mathrm{H} \beta$ line with a measured equivalent width (EW) of $E W_{\mathrm{H} \beta, *}=3.8 \AA$. We also find a faint He I $4471 \lambda$ line with an $E W_{\mathrm{HeI}}=1.3 \AA$ (see also Fig. 3). Similar results are obtained from the MaNGA data of the H II-5 region. In particular, we detect a faint $\mathrm{He}$ I $4471 \lambda$ line in the spectrum of the SLSN region with $E W_{\mathrm{HeI}}=0.7 \AA$. Since the presence of helium is an indicator of the young age of the underlying stellar population, we estimate the age following the method underlined in González Delgado et al. (1999); this method assumes an instantaneous burst of star formation $\left(10^{6} M_{\odot}\right)$ at solar metallicity, with an initial mass function modelled with a power-law function using a decay rate of $\alpha=-2.35$ and with mass cut-offs at $M_{\text {low }}=1 M_{\odot}$ and $M_{\text {up }}=80 M_{\odot}$. With these parameters we obtain an estimate for the stellar age of around $7 \mathrm{Myr}$; for lower metallicities we obtain similar results.

For an additional age estimate we use the stellar population synthesis code presented in Levesque \& Leitherer (2013), which gives age estimates related to the $\mathrm{H} \alpha \mathrm{EWs}$. The EW values for $\mathrm{H}$ II regions 1-3 are lower by a factor of 5 to 10 (for the galaxy centre) than the EWs measured for the brightest $\mathrm{H}$ II regions 5-7 (see Table 2), indicating a younger stellar age of the latter regions. Assuming a metallicity of $Z=0.008$ corresponding to $\sim 0.6 Z_{\odot}$, the derived stellar population ages for $\mathrm{H}$ II regions 5-7 are indeed younger $(\sim 6-7 \mathrm{Myr})$ than the H II regions in the rest of the galaxy ( $\geq 10-20 \mathrm{Myr})$. The SLSN region shows an intermediate value, implying that its stellar age is younger than the

\footnotetext{
3 The spectrum is available at https://dr12.sdss.org/
} spectrumDetail $? \mathrm{mjd}=52614$ \&fiber $=502 \&$ plateid=944 
Table 2. Energy fluxes (in units of $10^{-15} \mathrm{erg} / \mathrm{cm}^{2} / \mathrm{s}$ ) measured for the main emission lines identified in the spectra of each $\mathrm{H}$ II region.

\begin{tabular}{lcccccccc}
\hline \hline Region & Hbeta & [O III] 5007 & [N II] 6550 & Halpha & [N II] 6586 & [S II] 6718 & [S II] 6732 & $E W(\mathrm{H} \alpha)$ \\
\hline H II-1 & $1.63 \pm 0.05$ & $0.61 \pm 0.04$ & $0.80 \pm 0.04$ & $6.70 \pm 0.10$ & $2.39 \pm 0.06$ & $1.13 \pm 0.04$ & $0.78 \pm 0.04$ & 42.4 \\
H II-2 & $2.52 \pm 0.11$ & $0.61 \pm 0.10$ & $1.46 \pm 0.17$ & $11.45 \pm 0.27$ & $4.12 \pm 0.16$ & $1.72 \pm 0.0 .11$ & $1.25 \pm 0.14$ & 21.0 \\
H II-3 & $2.41 \pm 0.08$ & $0.58 \pm 0.05$ & $1.08 \pm 0.05$ & $10.13 \pm 0.25$ & $3.29 \pm 0.09$ & $1.48 \pm 0.04$ & $1.05 \pm 0.06$ & 41.9 \\
H II-4 & $1.71 \pm 0.05$ & $1.24 \pm 0.04$ & $0.59 \pm 0.12$ & $6.61 \pm 0.15$ & $1.87 \pm 0.04$ & $1.08 \pm 0.02$ & $0.74 \pm 0.02$ & 104.9 \\
H II-5 & $14.67 \pm 0.06$ & $14.83 \pm 0.31$ & $4.79 \pm 0.12$ & $52.10 \pm 0.80$ & $14.02 \pm 0.25$ & $7.72 \pm 1.42$ & $5.81 \pm 0.13$ & 189.4 \\
H II-6 & $1.39 \pm 0.03$ & $1.38 \pm 0.03$ & $0.44 \pm 0.02$ & $4.64 \pm 0.06$ & $1.32 \pm 0.03$ & $0.80 \pm 0.02$ & $0.56 \pm 0.01$ & 166.4 \\
H II-7 & $7.55 \pm 0.21$ & $8.10 \pm 0.20$ & $2.37 \pm 0.08$ & $27.34 \pm 0.61$ & $8.00 \pm 0.15$ & $4.26 \pm 0.08$ & $3.13 \pm 0.08$ & 139.3 \\
\hline
\end{tabular}

Table 3. Physical properties derived from the integrated spectra of each H II region.

\begin{tabular}{lcccccc}
\hline \hline Region & $\begin{array}{c}S F R \\
\left(M_{\odot} \mathrm{yr}^{-1}\right)\end{array}$ & $\begin{array}{c}s S F R / L / L^{*} \\
\left(M_{\odot} \mathrm{yr}^{-1}\right)\end{array}$ & $\begin{array}{c}\mathrm{N} 2 \\
12+\log (\mathrm{O} / \mathrm{H})\end{array}$ & $\begin{array}{c}\mathrm{O} 3 \mathrm{~N} 2 \\
12+\log (\mathrm{O} / \mathrm{H})\end{array}$ & $\log U$ & $\begin{array}{c}E(B-V) \\
(\mathrm{mag})\end{array}$ \\
\hline H II-1 & $0.26 \pm 0.01$ & $0.56 \pm 0.15$ & $8.54 \pm 0.16$ & $8.53 \pm 0.18$ & $-3.35 \pm 0.01$ & $0.37 \pm 0.03$ \\
H II-2 & $0.56 \pm 0.02$ & $0.73 \pm 0.15$ & $8.54 \pm 0.16$ & $8.57 \pm 0.18$ & $-3.58 \pm 0.01$ & $0.46 \pm 0.03$ \\
H II-3 & $0.41 \pm 0.01$ & $0.41 \pm 0.17$ & $8.52 \pm 0.16$ & $8.56 \pm 0.18$ & $-3.46 \pm 0.01$ & $0.41 \pm 0.01$ \\
H II-4 & $0.22 \pm 0.01$ & $0.42 \pm 0.14$ & $8.49 \pm 0.16$ & $8.45 \pm 0.18$ & $-3.35 \pm 0.01$ & $0.30 \pm 0.03$ \\
H II-5 & $1.43 \pm 0.02$ & $2.67 \pm 0.36$ & $8.48 \pm 0.16$ & $8.41 \pm 0.18$ & $-3.01 \pm 0.01$ & $0.22 \pm 0.03$ \\
H II-6 & $0.25 \pm 0.03$ & $0.96 \pm 0.23$ & $8.49 \pm 0.16$ & $8.42 \pm 0.18$ & $-3.10 \pm 0.01$ & $0.16 \pm 0.03$ \\
H II-7 & $0.78 \pm 0.02$ & $1.93 \pm 0.30$ & $8.50 \pm 0.16$ & $8.41 \pm 0.18$ & $-2.99 \pm 0.01$ & $0.24 \pm 0.03$ \\
\hline
\end{tabular}

Notes. The table lists: (Col. 2) the star formation rate and (Col. 3) the specific star formation rate per unit luminosity; (Cols. 4 and 5$)$ the metallicity obtained with the $\mathrm{N} 2$ and the O3N2 indicators; (Col. 6) the ionisation parameter log $U$ estimated using the [S II] lines and the formulation given in Díaz et al. (2000), and (Col. 7) the estimate of the extinction $E(B-V)$.

adjacent regions but older than the most active ones. This evidence is also in agreement with the detection of helium at the SLSN site.

Subsequently, we corrected our PMAS H II spectra for the underlying stellar populations using the stellar models provided by the SED@ code as templates (Cerviño \& Luridiana 2004). These models consider a Salpeter initial mass function in the mass range $0.1-120 M_{\odot}$ and use the Padova isochrone tables (Girardi et al. 2002) for an age of $7 \mathrm{Myr}$ for regions 5, 6, and 7, and an age of $15 \mathrm{Myr}$ for the remaining regions. Given the better spatial resolution of PMAS data, we used the segmentation map derived from PMAS to extract $\mathrm{H}$ II region spectra from MaNGA data. We measured the emission line fluxes with the SHerpa IFU line fitting software (SHIFU; García-Benito, in prep.), based on CIAOs SHERPA package (Freeman et al. 2001; Doe et al. 2007) by fitting single Gaussians to residual spectra obtained after subtracting the STARLIGHT fit from the observed spectrum (see description below). A first-order polynomial was used to fit the continuum baseline in order to take into account small deviations of the fit. Measuring the emission lines in the residual spectra, particularly Balmer and Paschen lines, corrects their fluxes affected by the presence of absorption wings of stellar origin. The list of the main emission lines identified in the spectra of each region is shown in Table 2, while in Table A.1 we list additional line measurements.

After correcting the data sets we finally derive several spatially-resolved physical properties of the host galaxy. We determine the SFR using the $\mathrm{H} \alpha$ line flux $F_{\mathrm{H} \alpha}$ diagnostic (Kennicutt 1989), where $\operatorname{SFR}\left[M_{\odot} \mathrm{yr}^{-1}\right]=7.9 \times 10^{-42} 4 \pi d_{l}^{2} F_{\mathrm{H} \alpha}$ ( $d_{l}$ is the luminosity distance of the galaxy). The SFR in each spaxel is shown in Fig. 4. We find a low value for the SFR of the $\mathrm{H}$ II region at the SLSN location of $S_{F R}=0.22 \pm 0.01 M_{\odot} \mathrm{yr}^{-1}$, while the highest values are found for the bright and younger regions 5 and $7: S F R_{5}=1.43 \pm 0.02 M_{\odot} \mathrm{yr}^{-1}$ and $S F R_{7}=0.78 \pm$ $0.02 M_{\odot} \mathrm{yr}^{-1}$ (see also Table 3). The integrated SFR for the part of the galaxy covered by our observations is $\sim 4.0 \pm 0.07 M_{\odot} \mathrm{yr}^{-1}$.
Assuming that the remaining part of the galaxy, which is smaller in area than that covered by our observations, shows a similar value, we estimate an integrated SFR for the total galaxy of $\sim 7 M_{\odot} \mathrm{yr}^{-1}$ in line with the SFR inferred from the GALaxy Evolution Explorer (GALEX) near-UV data by Nicholl et al. (2017). The authors of this paper also claim that $\sim 70 \%$ of the total SFR in the galaxy is obscured and obtain an estimate of the total SFR using additional archival measurements in the infrared, X-ray, and radio of $S F R_{\mathrm{tot}} \simeq 15 M_{\odot} \mathrm{yr}^{-1}$. Our estimate is in agreement with their results.

Furthermore, we compute the specific SFR (sSFR), i.e. the star formation rate per unit luminosity $\left(s S F R=S F R /\left(L / L^{*}\right)\right)$. We use the method described in Christensen et al. (2004), where a map of the rest-frame $B$-luminosity is weighted with the SFR map obtained above. The $B$-band rest-frame magnitude is derived from archival $g^{\prime}$-band Pan-STARRS images, which are almost identical to the rest-frame $B$-band wavelength range, and from this estimate the luminosity is obtained by computing the corresponding absolute magnitude after projecting the PMAS data onto the Pan-STARRS image. Finally, we multiply the SFR map with the ratio between the absolute $B$-magnitude map and the magnitude of a $M_{B}=-20.1$ galaxy (an average value that has been inferred for the break in the Schechter luminosity function at redshift $z=0.04$ and $z=0.07$ from the analysis of blue galaxies in the DEEP2 and COMBO-17 surveys, Faber et al. 2007). The results are shown in Fig. 4.

The peculiarity of the host of SN 2017egm is underlined by its placement in the BPT diagram (Baldwin et al. 1981), which is a diagnostic based on emission-line flux ratios, and is used to distinguish star-forming galaxies from active galactic nuclei (AGN) like Seyferts and LINERs. The location on the starforming branch is also an indicator of the ionisation field of the gas, with large ionised H II regions found in the upper left region of the BPT diagram (Sánchez et al. 2015). We use the emission-line fluxes measured in the integrated H II regions to determine their respective locations in the BPT diagrams. For 

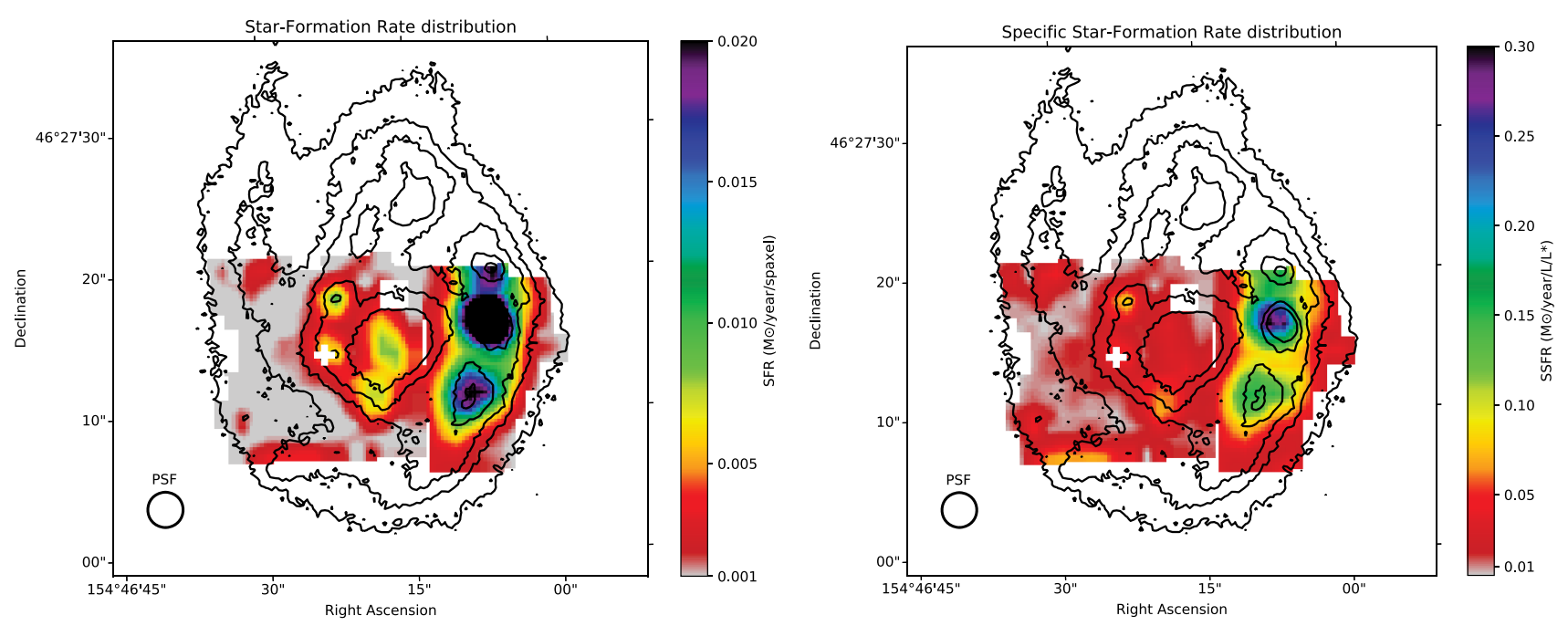

Fig. 4. Left panel: SFR map in the galaxy for the two fields covered by PMAS observations, computed using the Kennicutt (1989) diagnostic. Right panel: specific SFR obtained by weighting the SFR map and the rest-frame B-luminosity map, applying the same method described in Christensen et al. (2004). The dimensions of each spaxel have been interpolated in the figure, showing elements of $0.25 \times 0.25$ arcsec. The SLSN position is marked with a white cross.

comparison, we include two large samples of SLSN host galaxies from Leloudas et al. (2015) and Perley et al. (2016) distinguishing H-poor (Type I) and H-rich (Type II) SLSNe.

As shown in Fig. 5, all H II regions of NGC 3191 analysed in this paper are located in the lower part of the star-forming branch of the BPT diagram, where metal-rich and low-ionisation $\mathrm{H}$ II regions and the bulk of the general SDSS galaxy population are found. The location of SLSN 2017egm is one of the lowest on the star-forming side of the BPT diagram, implying that SLSN 2017egm is one of the most metal-rich H-poor SLSN found to date, more similar to the environment of H-rich SLSNe.

For the estimate of other physical properties we used PYNEB which computes the physical conditions of H II nebulae from specific diagnostic line ratios. For the estimate of the metallicity we applied two methods. First, we used two emissionline indicators with the calibrations provided in Marino et al. (2013): 1) the $\mathrm{N} 2$ method defined as $12+\log (\mathrm{O} / \mathrm{H})=8.743+$ $0.462 \times \log ([\mathrm{N}$ II $] 6584 / \mathrm{H} \alpha)$, and 2$)$ the O3N2 method defined as $12+\log (\mathrm{O} / \mathrm{H})=8.533-0.214 \times \log ([\mathrm{O}$ III $] 5007 / \mathrm{H} \beta \times$ $\mathrm{H} \alpha /[\mathrm{N}$ II $] 6584)$. The results for each $\mathrm{H}$ II region are reported in Table 3. Thanks to the presence of auroral lines in some of the spectra, we were also able to obtain elemental abundances obtained by means of the "direct" method (see Table A.3). Both methods indicate a higher metallicity for the centre of the galaxy $\left(Z_{2}=0.7 Z_{\odot}\right.$, with $Z_{\odot}=8.69$, Asplund et al. 2009), while the SLSN site shows slightly lower metallicities $\left(Z_{4}=0.6 Z_{\odot}\right)$ similar to the bright $\mathrm{H}$ II regions in the western part of the galaxy. In general, the metallicity distribution in the galaxy shows a decreasing distribution toward the external bright $\mathrm{H}$ II regions (see also Fig. 6), but has some asymmetry between the western and the eastern spiral arms.

We note that Nicholl et al. (2017) and Bose et al. (2017) find a much higher value for the metallicity using the BOSS spectrum of the galaxy centre of 2.0 and $1.6 Z_{\odot}$, respectively. All these estimates use distinct R23-based metallicity indicators to estimate the metallicity, which is known, however, to give overestimates of $Z$ (Kennicutt et al. 2003; Yin et al. 2007). In fact, from the same spectra we obtain a metallicity of $0.7 Z_{\odot}$ using the N2 parameter from Marino et al. (2013). Our estimate is not only based on the most updated calibrations available, but it is also supported by the spectral energy distribution modelling made in Nicholl et al. (2017). Chen et al. (2017b) find a high metallicity value $\left(1.3 Z_{\odot}\right)$ using the older calibration (Pettini \& Pagel 2004) of the $\mathrm{O} 3 \mathrm{~N} 2$ indicator. However, they use the same analysis developed by Bianco et al. (2016), which consists in checking a set of different metallicity indicators, and indeed find lower values when considering the most updated calibrations, in agreement with our results.

We also use the [S II] doublet to estimate the ionisation using the formulation given in Díaz et al. (2000), obtaining that the H II-5, 7 regions show a higher degree of ionisation than the other regions, including the one at the centre of the galaxy (see Table 3 ). In addition to other methods, [S II] lines have also been used to estimate the electron density and temperatures of the gas in H II regions. In general we find electron density values $\left(\sim 100 \mathrm{~cm}^{-3}\right)$ and temperatures $\left(T_{\mathrm{e}} \sim 10000 \mathrm{~K}\right)$ typical of $\mathrm{H} \mathrm{II}$ regions, although for the central regions we observe lower values $\left(T_{\mathrm{e}, 2} \sim 7500 \mathrm{~K}\right)$.

\section{Stellar populations and kinematics shaped by an interaction with its neighbour?}

NGC 3191 is not an isolated galaxy. It has a companion, MCG+08-19-017, at a projected distance of $\sim 45 \mathrm{kpc}$ (between the galaxy centres) and a radial velocity difference of only $\sim 200 \mathrm{~km} \mathrm{~s}^{-1}$. A colour picture of the two galaxies is shown in Fig. 7. From the analysis of the SDSS spectra described in Sect. 2, we find that MCG+08-19-017 has a low SFR of $0.15 M_{\odot} \mathrm{yr}^{-1}$, and an older stellar population as shown by a low $\mathrm{H} \alpha$ EW of only $21 \AA$ and a significant stellar absorption component in the $\mathrm{H} \beta$ line (EW of $6.3 \AA$ ). The metallicity is similar to the values observed in the centre of NGC 3191 with $Z(N 2) \sim 0.7 M_{\odot}$ using the calibrations of Marino et al. $(2013)^{4}$. No IFU data are available for the companion.

The somewhat disturbed morphology of NGC 3191 suggests that the two galaxies could have interacted gravitationally, which

\footnotetext{
4 The SDSS spectrum of the galaxy companion can be found at https: //dr12.sdss.org/spectrumDetail?mjd=52614\&fiber= $505 \&$ plateid $=944$
} 


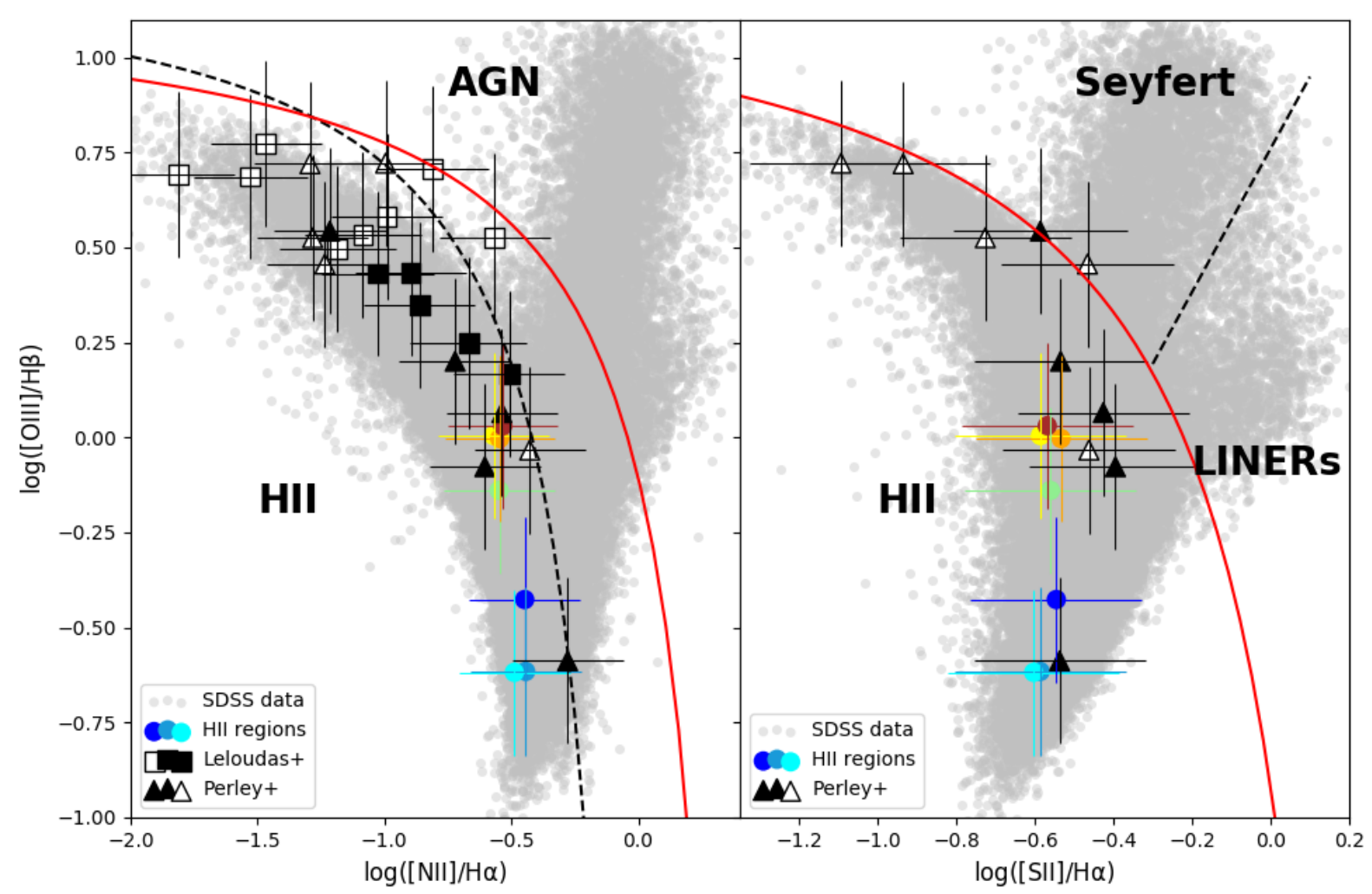

Fig. 5. BPT emission-line diagnostic used to distinguish the ionisation mechanism acting in the nebular gas in galaxies. Two distinct diagnostics are used here: [O III] $5008 \AA / \mathrm{H} \beta$ versus [N II] $6584 \AA$ $/ \mathrm{H} \alpha$ (left) and the [S II] 6718/32 A doublet instead of [N II] (right). The solid red curves in both panels denote the theoretical demarcation line between AGNs and star-forming galaxies proposed by Kewley et al. (2001). In the left panel, the black dashed curve corresponds to the empirical demarcation between AGNs and SF galaxies proposed by Kauffmann et al. (2003), while in the right panel the black dashed line marks the division between active Seyfert galaxies and LINERs proposed by (Kewley et al. 2006). Grey data points are taken from the SDSS DR7 sample of galaxies described in Tremonti et al. (2004). Coloured circles correspond to the H II regions described in the text with the same colour-coding used in Fig. 1. Squares and triangles represent SLSN-host data from Leloudas et al. (2015) and Perley et al. (2016); empty symbols represent H-poor SLSNe, filled symbols H-rich SLSNe. The SLSN 2017egm location (light green circle) shows it is one of the most metal-rich Type I SLSN.

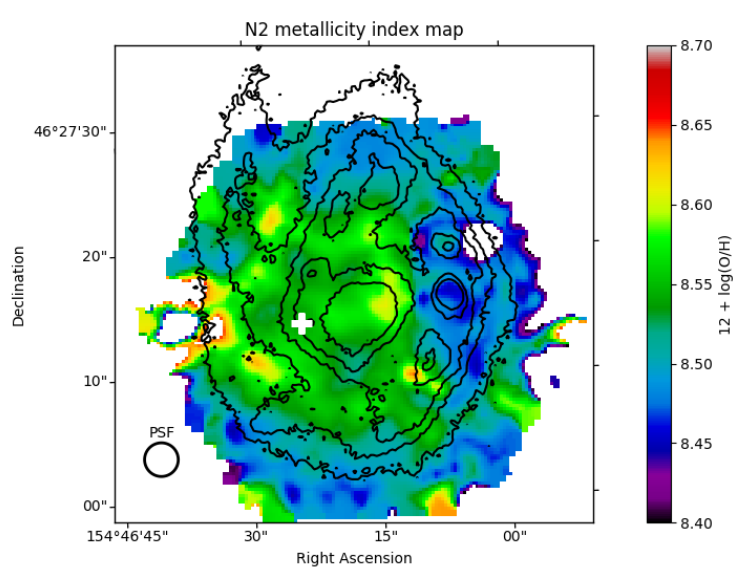

Fig. 6. Metallicity map obtained from MaNGA data using the N2 indicator as given by Marino et al. (2013). The location of SLSN 2017egm is indicated by a black cross, while the location of PTF10bgl is indicated by a black circle.

would explain the observed distribution of SFR and metallicity in NGC 3191. In fact, MCG+08-19-017 also shows more intense star formation on its eastern side, in the direction towards

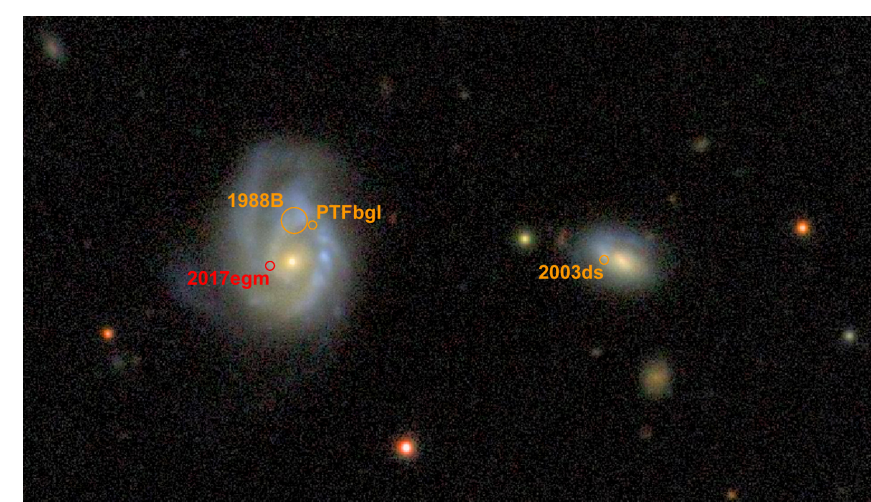

Fig. 7. Colour image of NGC 3191 and its neighbour MCG+08-19-017 created combining multiband data from the PanSTARRS and the SDSS image catalogues. Also marked are the locations of the four SNe that occurred in this galaxy pair. North is up and east is to the left; the field of view is $3.2 \times 1.8$.

NGC 3191, suggesting that there had been a near pass-by of the two galaxies which could have compressed the gas on their facing sides as a result of the closest encounter. In order to investigate the kinematics in NGC 3191, we use the observations 

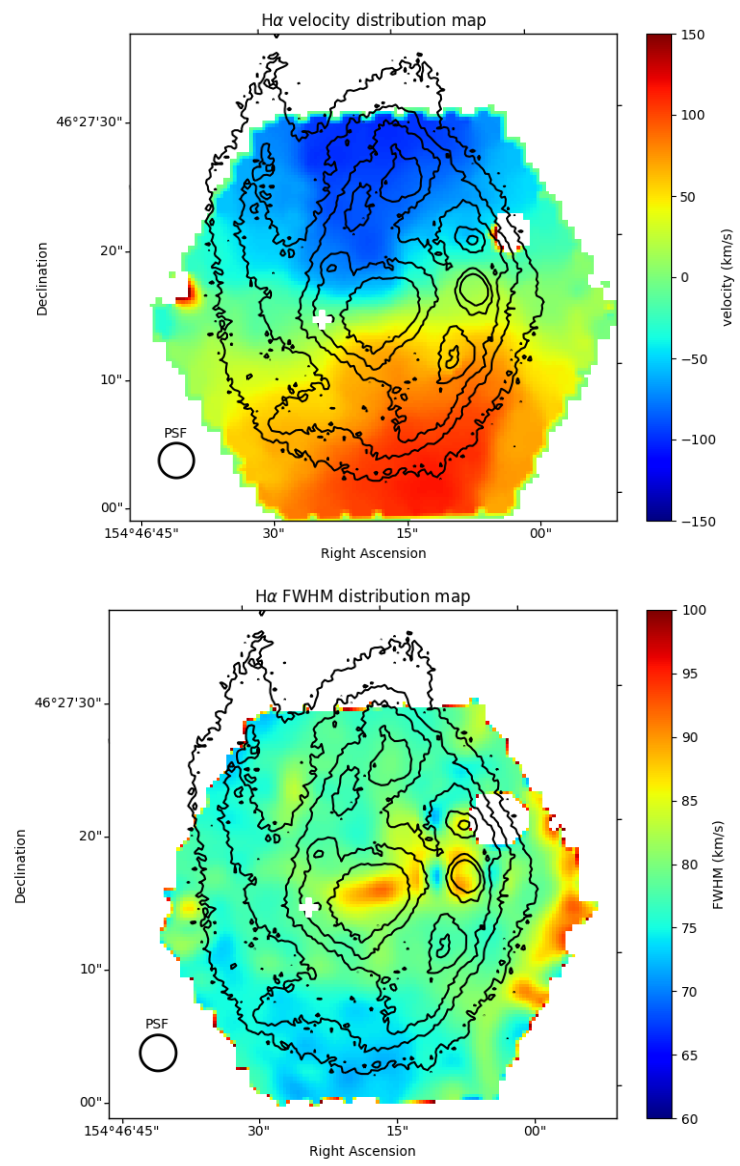

Fig. 8. Upper panel: velocity field map of the gas in NGC 3191 with kinemetric ellipses overplotted as dashed lines. Lower panel: FWHM $\mathrm{H} \alpha$ distribution map.

available from the MaNGA survey. In Fig. 8 we plot the velocity field determined from fitting the $\mathrm{H} \alpha$ line with a Gaussian and taking the peak and FWHM for each spaxel. The velocity field of the host is surprisingly regular considering its morphology, only the outer parts of the disk seem to be somewhat twisted. The widths of the lines only show an enhancement in the central region and within the brightest of the three large SF regions in the west. Line broadening in the centre is expected as it probes a higher amount of gas in the bulge. The enhancement in an active $\mathrm{SF}$ region is a reflection of turbulence due to the ongoing heavy SF.

To quantify disturbances in the velocity field we perform a kinemetric analysis following the method described in Krajnović et al. (2006) and plot the results in Fig. 9. From the ratio of $\mathrm{k} 5 / \mathrm{k} 1$ we see that there is no strong disturbance in a smooth velocity field which would lead to much higher values for $\mathrm{k} 5$ (the first higher order term in the analysis). However, the ellipticity parameter $(q)$ increases from near circles (as expected for a nearly face-on galaxy) in the centre to more elongated ellipses in the outer parts of the host, while in undisturbed galaxies the ellipticity is expected to be constant. This could be a sign of interaction with its neighbour, introducing a tangential or warplike disturbance in the velocity field.

Considering the evidence for interaction and the rather atypical location of the SLSN next to the centre, we also perform a thorough analysis of the stellar population in the galaxy. For this we use the STARLIGHT code (Cid Fernandes et al. 2005), which estimates the properties of the stellar population from

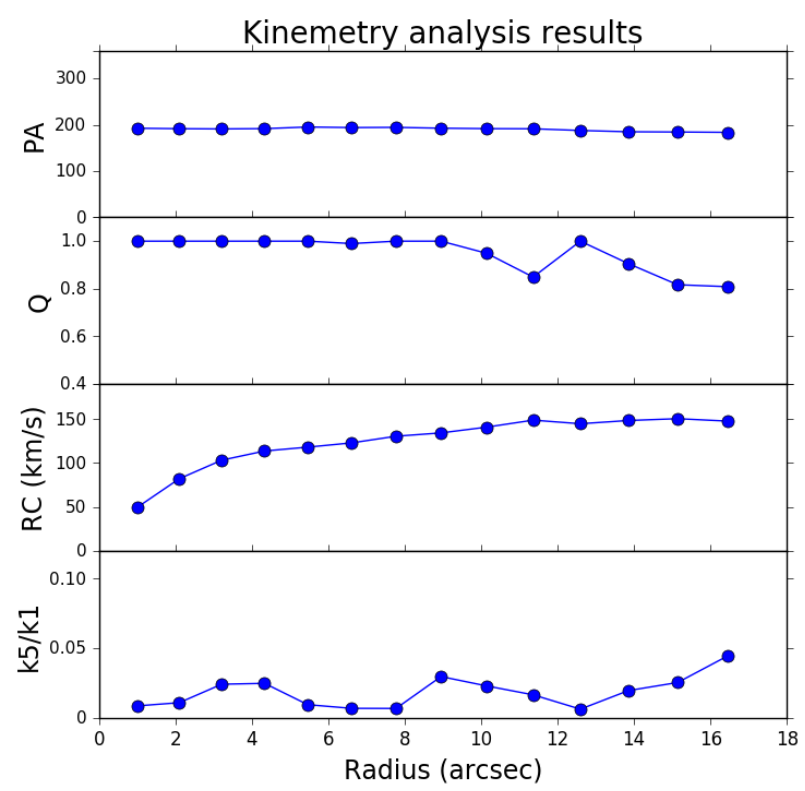

Fig. 9. Best-fit parameters obtained from the kinemetric analysis (Krajnović et al. 2006) for different positions of the tilted rings relative to the centre of NGC 3191. From the top: position angle in degrees, the ellipticity parameter q, the rotation curve (RC) corresponding to the first-order $k 1$ of the harmonic expansion corrected for the inclination of the galaxy, and the $k 5 / k 1$ ratio.

the observed spectral energy distribution, and using the stellarpopulation synthesis models given in Bruzual \& Charlot (2003). We use the spectra of the H II regions extracted from the MaNGA datacube, which have better spectral resolution and a wider wavelength range. The total stellar masses and distributions of the stellar mass with age for each $\mathrm{H}$ II region are listed in Table 4. In Fig. 10 we show the detailed fitting and stellar population distribution for three particular regions: 1) the SLSN region; 2) the region located at the galaxy centre (H II-2); and 3 ) the $\mathrm{H}$ II-5 region, currently the most active SF region in the galaxy.

We note that H II regions characterised by the youngest ages (weighted by their luminosities) show the lowest metallicities. This becomes clear when looking at the distribution of the age weighted by the luminosity in the galaxy $y^{5}$ in Fig. 11, with the distribution of the metallicity estimated with the $\mathrm{N} 2$ indicator (see Fig. 6). Our detailed analysis of the stellar populations shows the presence of at least two distinct stellar populations in all $\mathrm{H}$ II regions, one spanning from 1-10 Gyr, which contributes almost all of the stellar mass, and an additional very young population (1-10 Myr), see also Table 4. While the high luminosity of the most active SF regions on the western side of the galaxy is due to an additional very young population of around $1 \mathrm{Myr}$ (see also the lower panel in Fig. 11), the galaxy centre is dominated by the old stellar population $(>1 \mathrm{Gyr})$ with only very low SF activity in the past tens of Myrs (middle panel in Fig. 11). The observed light from SF regions close to the centre including the SLSN site have an older population at the lower end of the initial SF activity at $\sim 1$ Gyr down to several 100 Myr ago, but additional new activity started at $10 \mathrm{Myr}$ and extends down to 2-4 Myr. A stellar population of 3-10 Myr would correspond to the evolution time of a massive stars of $20-60 M_{\odot}$ until explosion

5 We run STARLIGHT on the MaNGA cube using the same SSPs ingredients as the H II spectra. The output was packed and processed with the Pycasso pipeline (Cid Fernandes et al. 2013; de Amorim et al. 2017). 
Table 4. Physical properties for each H II region from STARLIGHT.

\begin{tabular}{|c|c|c|c|c|c|c|}
\hline Region & $\begin{array}{c}M_{*} \\
\left(\log M_{\odot}\right) \\
\end{array}$ & $\begin{array}{c}\text { Age } \\
(\log [\mathrm{yr}])\end{array}$ & $\begin{array}{c}f(\text { age }<30 \text { Myr }) \\
(\%)\end{array}$ & $\begin{array}{c}f(30 \mathrm{Myr}<\text { age }<\mathrm{Gyr}) \\
(\%)\end{array}$ & $\begin{array}{c}f(\text { age }>\text { Gyr }) \\
(\%)\end{array}$ & $\begin{array}{c}f(L<30 \mathrm{Myr}) \\
(\%)\end{array}$ \\
\hline H II-1 & 8.76 & 8.43 & 1.4 & 37.9 & 60.7 & 23.9 \\
\hline H II-2 & 9.54 & 8.43 & 0.4 & 16.7 & 82.9 & 14.5 \\
\hline H II-3 & 9.12 & 8.69 & 0.9 & 24.3 & 74.8 & 20.7 \\
\hline H II-4 & 8.28 & 8.24 & 2.1 & 42.2 & 55.7 & 35.0 \\
\hline H II-5 & 8.86 & 7.93 & 3.5 & 10.5 & 86.0 & 47.4 \\
\hline H II-6 & 7.83 & 7.81 & 4.3 & 30.1 & 65.6 & 47.1 \\
\hline H II-7 & 8.77 & 8.07 & 3.1 & 36.5 & 60.4 & 38.5 \\
\hline
\end{tabular}

Notes. The table lists the total stellar mass (Col. 2); the light-weighted age (Col. 3); the mass fraction of stellar populations younger than $30 \mathrm{Myr}$, between $30 \mathrm{Myr}$ and $1 \mathrm{Gyr}$, and older than $1 \mathrm{Gyr}$ (Cols. 4-6); and finally the fraction in luminosity of stellar populations younger than $30 \mathrm{Myr}$ (Col. 7).

(see e.g. Kuncarayakti et al. 2013). The fraction in luminosity of stars younger than $30 \mathrm{Myr}$ is high for $\mathrm{H}$ II regions 4-7, but these regions are also characterised by low metallicity, see Table 3. Consequently, we infer that the presence of these young stars is responsible for the observed lower metallicity in these regions, and that these young and massive star populations are characterised by an intrinsically lower metal abundance $\left(<0.6 Z_{\odot}\right)$ than the entire host, including the progenitor of SLSN 2017egm.

All H II regions show a SF peak around 10-15 Myr. At that time, a burst of star formation very likely involved a significant galaxy. These metal-poor young populations could very well come from an interaction with the neighbouring galaxy, leading to compression and shocks of the gas in the part of the host facing the companion. Possibly, the same process lead to enhanced star formation on the eastern part of the neighbouring galaxy. The slightly "older" regions of this starburst around the centre of NGC 3191 maintain the information on the burst of star formation caused by this gravitational interaction. The SLSN progenitor could be the first product of this prolonged burst of star formation (a few Myr), while the recently triggered SF in the western H II regions are still too young to actually host any $\mathrm{SNe}$. In this picture, the star formation would still be taking place with older metal-enriched gas, hence still posing a problem if the SLSN has to be in a metal-poor environment. Such interactions, however, can also funnel metal-poor H I gas into the central regions of a galaxy, thereby giving rise to a SLSN in a metal-rich environment. Another possibility is the transfer of metal-poor gas from the interacting galaxy to NGC 3191. However, the spectrum of the centre of MCG+08-19-017 shows a metallicity value similar to that of NGC 3191, making this possibility less likely. Instead of a direct gas transfer, pristine gas from possible residuals of former dwarf companions ripped apart by the interaction could also be transferred into the main galaxy. Resolved HI data of both galaxies could give some clues about this scenario (Michałowski et al. 2015).

\section{Locations of SN 1988B, SN 2003ds, and PTF10bgl}

The galaxy pair NGC 3191 and MCG+08-19-017 have hosted SN 2017egm and another three SNe in the past two decades. This emphasises the increased SF and SN activity due to mutual interaction in both galaxies discussed in the previous section. In Fig. 7 we plot the locations of all four SNe in the galaxy pair.

SN 1988B was a Type Ia SN discovered by P. Wild (Kosai et al. 1988) and classified as a SN Ia (Filippenko et al. 1988). Unfortunately, there are no precise coordinates reported in the literature, but Kosai et al. (1988) give the location as 10'”0 north of the galaxy centre. Cappellaro \& Turatto (1988) also mention the presence of $\mathrm{H} \alpha$ emission on top of the SN spectrum, indicative of a SF region at the $\mathrm{SN}$ site. SNe Ia can occur in any kind of galaxy and require an older population (generally from $2 \times 10^{8}$ to $10^{10}$ years, (Maoz \& Mannucci 2012) ) for the white dwarf progenitor to form. NGC 3191 has a large underlying older population with ages of 1-10 Gyr, hence the occurrence of a SN Ia in this galaxy is not surprising.

SN 2003ds was of Type Ic and took place in the neighbouring galaxy. Its location near the bright SF region to the west which we attribute to the mutual interaction between the two galaxies closely matches expectations: Type Ic $\mathrm{SNe}$ are presumably Wolf-Rayet stars that have lost their $\mathrm{H}$ and $\mathrm{He}$ envelope through winds or smaller outbursts (e.g. Georgy et al. 2009; Tramper et al. 2015), hence the original progenitor must have been a rather massive star $\left(>25-30 M_{\odot}\right)$. An alternative is a binary progenitor where the companion has removed the envelope of the star, leaving a relatively small progenitor star. By studying the age of surrounding HII regions, Kuncarayakti et al. (2013) found a preference for young ages and therefore the single-star scenario, albeit with some exceptions. No reliable Type Ic progenitor detection has been made to date. Ic SNe presumably require near solar metallicity which facilitates the strength of stellar winds (see e.g. Leloudas et al. 2011; Modjaz et al. 2011; Kuncarayakti et al. 2013), but the statistical significance of the results are controversial (Leloudas et al. 2011). Moreover, all agree that the metallicities are higher than those found for GRB (and hence SLSN-I) environments. MCG+08-19-017 indeed has a similar metallicity to that of the core of NGC 3191; however, the Legacy Survey spectra only probe the central part of the galaxy and not the SF region of $2003 \mathrm{ds}$. Since there are no IFU data on this galaxy, we cannot draw any further conclusions on the properties of the progenitor site at this point.

PTF10bgl is a Type II SN (Arcavi et al. 2010; Bose et al. 2017), located just north of the three major SF regions in the west in the same spiral arm, but in a gap between SF regions. Fortunately, this region is also covered by the MaNGA data. The metallicity at the site of PTF10bgl is surprisingly similar to that of the site of $2017 \mathrm{egm}$ with $12+\log (\mathrm{O} / \mathrm{H})=8.52 \pm 0.18(\mathrm{~N} 2 \mathrm{pa}-$ rameter) and a log age of 8.32 yr. The location of PTF10bgl, however, is outside any active H II region in the host galaxy, which is reflected in a much lower SFR measured at the SN site: $S F R=0.05 \pm 0.01 M_{\odot} \mathrm{yr}^{-1}$.

\section{Conclusions}

In this work we present IFU data of a large part of the SLSN2017egm host galaxy, NGC 3191, which is the closest Type I SLSN discovered to date and has hosted another two SNe in the 

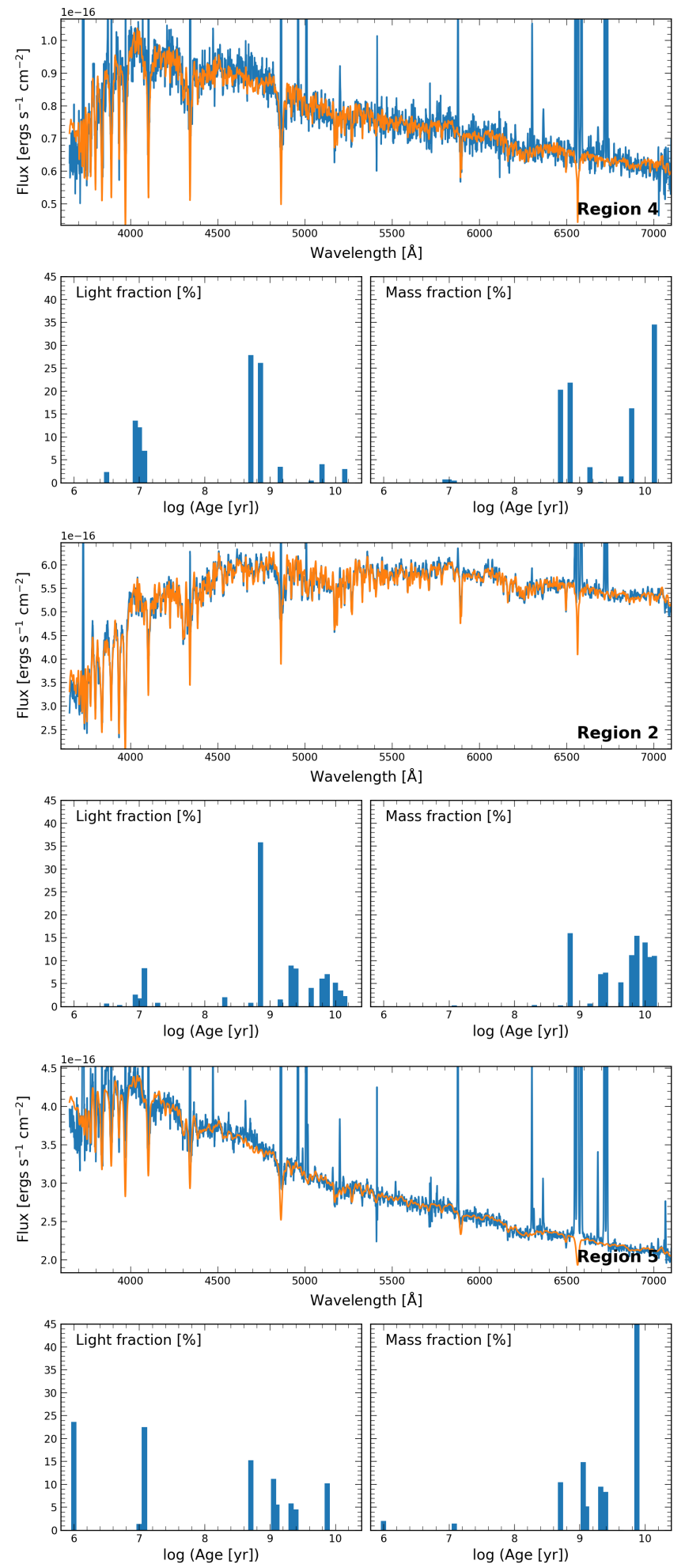

Fig. 10. From top to bottom, the stellar population analysis of H II regions 4 (the SLSN region), 2 (the galaxy centre), and 5 (the most active region in the galaxy). The upper panels show the fit to the observed spectral energy distributions, while the lower panels show the fraction in luminosity of stellar populations with different ages (left) and their contribution to the total stellar mass (right).

past. NGC 3191 is a large spiral galaxy with an integrated stellar mass of $M_{*}=10^{10.7} M_{\odot}$. The galaxy has an average metallicity of $\sim 0.6 Z_{\odot}$; it has a typical negative gradient distribution towards the external regions, as is usually found in bright large spirals.

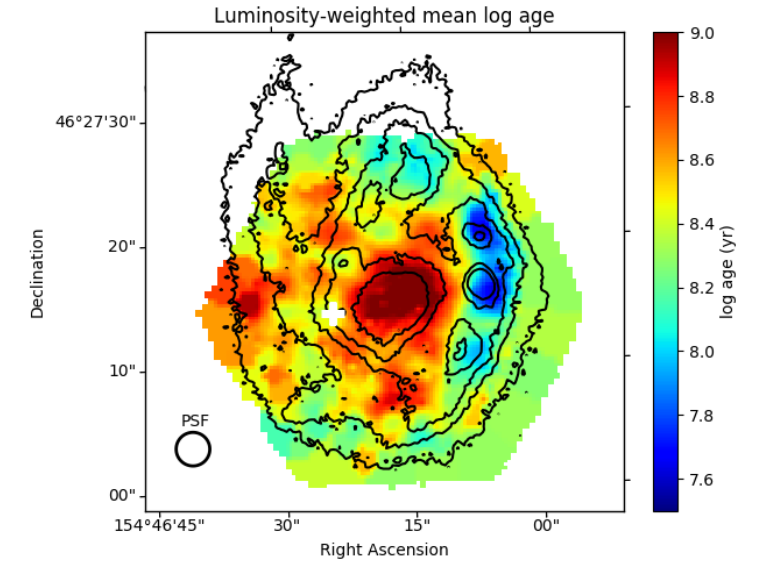

Fig. 11. Luminosity weighted (log) age distribution estimated with the STARLIGHT code, following the prescriptions given in Cid Fernandes et al. (2013). The location of SLSN 2017egm is reported with a black cross while the location of PTF10bgl is reported with a black circle.

However, these galaxies do not represent the canonical hosts for Type I SLSNe, which are usually low-mass star-bursting dwarf galaxies (Leloudas et al. 2015; Schulze et al. 2016; Perley et al. 2016; Cikota et al. 2017). The H II region around the SLSN, with a size of $\sim 1 \mathrm{kpc}$, has one of the highest metallicity values $\left(Z_{4}=0.6 Z_{\odot}\right)$ among the known Type I SLSNe to date, and higher than the proposed cut-off for Type I SLSNe progenitors of $0.4-0.5 Z_{\odot}$ (Schulze et al. 2016; Chen et al. 2017c). The only other IFU study of a SLSN-I host by Cikota et al. (2017) also shows that the SLSN are not coincident with the lowest metallicity (and highest SFR) region, but the value at the site of PTF11hrq $(12+\log (\mathrm{O} / \mathrm{H}) \sim 8.2)$ is still far below the value we find for the site of SN 2017egm. Our metallicity value is based on the most recent calibration of the $\mathrm{N} 2$ and $\mathrm{O} 3 \mathrm{~N} 2$ indicators by Marino et al. (2013). Taking other less reliable but frequently used calibrations in the literature for comparison (see Table A.2), we consistently find higher metallicities for the SLSN region. We also used the S23 indicator, which uses the bright doublet lines of [S III] 9069-9523 $\lambda \lambda$ in the parametrisation given by Pérez-Montero \& Díaz (2005). These lines are among the brightest in the H II regions, also at high metallicities, and do not suffer large extinction effects ${ }^{6}$.

SLSN 2017egm is not closely tracing the SF in its host as is usually the case for Type I SLSNe (Lunnan et al. 2015) and also for GRBs and their associated broad-line Type Ic SNe (Fruchter et al. 2006; Blanchard et al. 2016; but see the resolved study of PTF11hrq by Cikota et al. 2017). The SFR value measured at the SLSN site from the $\mathrm{H} \alpha$ flux $\left(S F R_{4}=0.22 \pm 0.01 M_{\odot} \mathrm{yr}^{-1}\right)$ is similar to values found for the integrated spectra of other SLSN hosts. In addition, SLSN 2017 egm did not explode in the youngest region of its host. Our stellar population analysis shows the presence of two main stellar populations with ages of $>500 \mathrm{Myr}$ for the older population, and around $10 \mathrm{Myr}$ for the younger population. In the SLSN region, the bulk of the observed luminosity (54\%) comes from an older population of stars, but a conspicuous fraction (35\%) originates from a young stellar population $(<30 \mathrm{Myr})$. In the bright SF regions in the western part of the galaxy a small fraction of stars (3\%) show an age younger than 10 Myr. Additional evidence from other indicators, such as the observed EWs of Balmer lines

6 See García-Benito et al. (2010) for the first map of this parameter using IFU data. 
and He I 4471 $\lambda \AA$, suggests the presence of stars with an age of the order of $\sim 10 \mathrm{Myr}$ and possibly younger. This value is higher than the value derived for the progenitor of PTF12dam (Thöne et al. 2015), which was possibly the youngest host to date, but similar to the age of PTF11hrq (Cikota et al. 2017). Still, this value is in line with the age of a rather massive star as required for current models of Type I SLSNe.

Star formation in this galaxy seems to have been at a low level for several hundred Myrs, after which it experienced a new recent burst in SF. Evidence for this is found in the brightest $\mathrm{H}$ II regions. Their location, in the apparent direction toward the companion galaxy MCG+08-19-017, could suggest a gravitational interaction which triggered the recent star formation. Kinematic analysis of NGC 3191 shows some deviations in the external regions of the galaxy with a "twist" in the velocity field that could very well be explained by an interaction within the last tens of Myrs. Evidence for an interaction can also be inferred from the metallicity distribution map (Fig. 6), where a negative gradient toward the western side of the galaxy (where the youngest H II regions are observed) is visible, but no such gradient exists on the eastern side. The same trend is visible in Fig. 2 of Chen et al. (2017b), where the authors use distinct R23-based metallicity indicators, that overestimate the metallicity. Rearrangement of gas in the galaxy, gas transfer, or the interaction causing metal-poor $\mathrm{H}$ I gas to be funneled into the galaxy could explain the occurrence of a SLSN Type I at low metallicity even in this solar-metallicity galaxy and indeed there is a small population of metal-poor stars present at the SLSN site. New SF activity due to interaction was also proposed for three other SLSN-I hosts (Cikota et al. 2017; Chen et al. 2017a).

NGC 3191 has previously hosted two other SNe, a Type Ia (SN 1988B) and a Type II SN (PTF10bgl), while the neighbouring galaxy MCG+08-19-017 hosted another Type Ic SN (SN 2003ds). PTF10bgl shares very similar physical properties of the environment with $2017 \mathrm{egm}$, such as gas metallicity and stellar age. The low SFR value is likely due to its location, which is at the border of the most active H II regions. We consider this as interesting constraints when trying to infer progenitor properties from environments considering that the two environments of different SN progenitors can be very similar.

Our study shows the importance of spatially resolved studies and detailed analysis of stellar populations to infer SNprogenitor properties. The detailed analysis of the spectra of different $\mathrm{H}$ II regions in the host allows us to understand the origin of the apparent metal anomaly in the SLSN environment. The implications of this result are several. Care must be taken when inferring characteristics of SN progenitors from the physical properties of their unresolved host galaxies. While it can provide valuable information in large statistical samples, it can be deceiving when used for individual cases. Moreover, a detailed analysis of the observed spectra is required, which takes into account the properties of the stellar population, not only those of the emitting gas. Finally, the use of powerful tools for studying galaxy kinematics are equally important to unveil traces of gravitational interactions that can trigger bursts of star formation. A global analysis is then necessary to precisely constrain the physical characteristics of very massive stars on their way to exploding as SLSNe.

Acknowledgements. We thank the referee for the constructive comments that have improved the paper. We also thank Steve Schulze and Yan Lin for their important comments to the paper. L.I., C.T., Z.C., A.d.U.P., and D.A.K. acknowledge support from the Spanish research project AYA2014-58381-P. C.T. and A.d.U.P. also acknowledge support from Ramón y Cajal fellowships RyC2012-09984 and RyC-2012-09975. D.A.K. and Z.C. acknowledge support from
Juan de la Cierva Incorporación fellowships IJCI-2015-26153 and IJCI-201421669. R.G.B. acknowledges support from the Spanish Ministerio de Economía y Competitividad, through projects AYA2016-77846-P and AYA2014-57490-P. L.I. wishes to thank Anna Serena Esposito for her kind availability and support in organising the figures presented in this paper.

\section{References}

Arcavi, I., Gal-Yam, A., Kasliwal, M. M., et al. 2010, ApJ, 721, 777

Asplund, M., Grevesse, N., Sauval, A. J., \& Scott, P. 2009, ARA\&A, 47, 481

Astropy Collaboration, Robitaille, T. P., Tollerud, E. J., et al. 2013, A\&A, 558, A33

Baldwin, J. A., Phillips, M. M., \& Terlevich, R. 1981, PASP, 93, 5

Bianco, F. B., Modjaz, M., Oh, S. M., et al. 2016, Astron. Comput., 16, 54

Blanchard, P. K., Berger, E., \& Fong, W.-f. 2016, ApJ, 817, 144

Bose, S., Dong, S., Pastorello, A., et al. 2017, ArXiv e-prints [arXiv: 1708.00864]

Bruzual, G., \& Charlot, S. 2003, MNRAS, 344, 1000

Cappellaro, E., \& Turatto, M. 1988, IAU Circ., 4549

Cerviño, M., \& Luridiana, V. 2004, A\&A, 413, 145

Chen, T.-W., Nicholl, M., Smartt, S. J., et al. 2017a, A\&A, 602, A9

Chen, T.-W., Schady, P., Xiao, L., et al. 2017b, ApJ, 849, L4

Chen, T.-W., Smartt, S. J., Yates, R. M., et al. 2017c, MNRAS, 470, 3566

Chomiuk, L., Chornock, R., Soderberg, A. M., et al. 2011, ApJ, 743, 114

Christensen, L., Hjorth, J., \& Gorosabel, J. 2004, A\&A, 425, 913

Cid Fernandes, R., Mateus, A., Sodré, L., Stasińska, G., \& Gomes, J. M. 2005, MNRAS, 358, 363

Cid Fernandes, R., Pérez, E., García Benito, R., et al. 2013, A\&A, 557, A86

Cikota, A., De Cia, A., Schulze, S., et al. 2017, MNRAS, 469, 4705

Dawson, K. S., Schlegel, D. J., Ahn, C. P., et al. 2013, AJ, 145, 10

de Amorim, A. L., García-Benito, R., Cid Fernandes, R., et al. 2017, MNRAS, 471, 3727

Delgado, A., Harrison, D., Hodgkin, S., et al. 2017, Transient Name Server Discovery Report, 591

Díaz, A. I., Castellanos, M., Terlevich, E., \& Luisa García-Vargas, M. 2000, MNRAS, 318, 462

Doe, S., Nguyen, D., Stawarz, C., et al. 2007, in Astronomical Data Analysis Software and Systems XVI, eds. R. A. Shaw, F. Hill, \& D. J. Bell, ASP Conf. Ser., 376, 543

Dong, S., Bose, S., Chen, P., et al. 2017, ATel, 1049

Drake, A. J., Djorgovski, S. G., Mahabal, A., et al. 2009, ApJ, 696, 870

Faber, S. M., Willmer, C. N. A., Wolf, C., et al. 2007, ApJ, 665, 265

Filippenko, A. V., Cohen, R. D., \& Junkkarinen, V. T. 1988, IAU Circ., 4535

Freeman, P., Doe, S., \& Siemiginowska, A. 2001, in Astronomical Data Analysis, eds. J.-L. Starck, \& F. D. Murtagh, Proc. SPIE, 4477, 76

Fruchter, A. S., Levan, A. J., Strolger, L., et al. 2006, Nature, 441, 463 Gal-Yam, A. 2012, Science, 337, 927

García-Benito, R., Díaz, A., Hägele, G. F., et al. 2010, MNRAS, 408, 2234

Georgy, C., Meynet, G., Walder, R., Folini, D., \& Maeder, A. 2009, A\&A, 502, 611

Girardi, L., Bertelli, G., Bressan, A., et al. 2002, A\&A, 391, 195

González Delgado, R. M., Leitherer, C., \& Heckman, T. M. 1999, ApJS, 125, 489

Hakobyan, A. A., Adibekyan, V. Z., Aramyan, L. S., et al. 2012, A\&A, 544, A81

Izzo, L., Thöne, C. C., Schulze, S., et al. 2017, MNRAS, 472, 4480

Kaiser, N., \& Pan-STARRS Team 2005, in BAAS, 37, 1409

Kauffmann, G., Heckman, T. M., Tremonti, C., et al. 2003, MNRAS, 346, 1055

Kennicutt, Jr., R. C. 1989, ApJ, 344, 685

Kennicutt, Jr., R. C., Bresolin, F., \& Garnett, D. R. 2003, ApJ, 591, 801

Kewley, L. J., Dopita, M. A., Sutherland, R. S., Heisler, C. A., \& Trevena, J. 2001, ApJ, 556, 121

Kewley, L. J., Groves, B., Kauffmann, G., \& Heckman, T. 2006, MNRAS, 372, 961

Kosai, H., Ikeya, K., Evans, R. O., et al. 1988, IAU Circ., 4533

Krajnović, D., Cappellari, M., de Zeeuw, P. T., \& Copin, Y. 2006, MNRAS, 366, 787

Kuncarayakti, H., Doi, M., Aldering, G., et al. 2013, AJ, 146, 30

Law, N. M., Kulkarni, S. R., Dekany, R. G., et al. 2009, PASP, 121, 1395

Law, D. R., Yan, R., Bershady, M. A., et al. 2015, AJ, 150, 19

Leloudas, G., Gallazzi, A., Sollerman, J., et al. 2011, A\&A, 530, A95

Leloudas, G., Schulze, S., Krühler, T., et al. 2015, MNRAS, 449, 917

Levesque, E. M., \& Leitherer, C. 2013, ApJ, 779, 170

Lunnan, R., Chornock, R., Berger, E., et al. 2014, ApJ, 787, 138

Lunnan, R., Chornock, R., Berger, E., et al. 2015, ApJ, 804, 90

Maoz, D., \& Mannucci, F. 2012, PASA, 29, 447

Marino, R. A., Rosales-Ortega, F. F., Sánchez, S. F., et al. 2013, A\&A, 559, A114

Michałowski, M. J., Gentile, G., Hjorth, J., et al. 2015, A\&A, 582, A78 
Modjaz, M., Kewley, L., Bloom, J. S., et al. 2011, ApJ, 731, L4 Nicholl, M., Berger, E., Margutti, R., et al. 2017, ApJ, 845, L8 Ofek, E. O., Cameron, P. B., Kasliwal, M. M., et al. 2007, ApJ, 659, L13 Pagel, B. E. J., Edmunds, M. G., Blackwell, D. E., Chun, M. S., \& Smith, G. 1979, MNRAS, 189, 95

Pastorello, A., Smartt, S. J., Botticella, M. T., et al. 2010, ApJ, 724, L16 Pérez-Montero, E., \& Díaz, A. I. 2005, MNRAS, 361, 1063

Perley, D. A., Quimby, R. M., Yan, L., et al. 2016, ApJ, 830, 13

Pettini, M., \& Pagel, B. E. J. 2004, MNRAS, 348, L59

Quimby, R. M., Aldering, G., Wheeler, J. C., et al. 2007, ApJ, 668, L99

Quimby, R. M., Kulkarni, S. R., Kasliwal, M. M., et al. 2011, Nature, 474, 487

Roth, M. M., Kelz, A., Fechner, T., et al. 2005, PASP, 117, 620

Sánchez, S. F., Pérez, E., Rosales-Ortega, F. F., et al. 2015, A\&A, 574, A47

Sandin, C., Becker, T., Roth, M. M., et al. 2010, A\&A, 515, A35
Schulze, S., Krühler, T., Leloudas, G., et al. 2016, MNRAS, in press ArXiv e-prints [arXiv: 1612.05978]

Smith, N., Li, W., Foley, R. J., et al. 2007, ApJ, 666, 1116

Soto, K. T., Lilly, S. J., Bacon, R., Richard, J., \& Conseil, S. 2016, MNRAS, 458, 3210

Stoll, R., Prieto, J. L., Stanek, K. Z., \& Pogge, R. W. 2013, ApJ, 773, 12

Taddia, F., Moquist, P., Sollerman, J., et al. 2016, A\&A, 587, L7

Thöne, C. C., de Ugarte Postigo, A., García-Benito, R., et al. 2015, MNRAS, 451, L65

Tonry, J. L., Stubbs, C. W., Kilic, M., et al. 2012, ApJ, 745, 42

Tramper, F., Straal, S. M., Sanyal, D., et al. 2015, A\&A, 581, A110

Tremonti, C. A., Heckman, T. M., Kauffmann, G., et al. 2004, ApJ, 613, 898

Yin, S. Y., Liang, Y. C., Hammer, F., et al. 2007, A\&A, 462, 535

York, D. G., Adelman, J., Anderson, Jr., J. E., et al. 2000, AJ, 120, 1579

\section{Appendix A: Additional tables}

Table A.1. Energy fluxes (in units of $10^{-15} \mathrm{erg} / \mathrm{cm}^{2} / \mathrm{s}$ ) measured for additional emission lines identified in each H II region spectrum.

\begin{tabular}{lccccccc}
\hline \hline Region & {$[\mathrm{O}$ II] 3727, 29 } & {$[\mathrm{Ne}$ III] 3869 } & {$[\mathrm{O}$ III] 4959 } & He I 5876 & [O I] 6300 & [O II] 7319, 30 & [S III] 9069, 9531 \\
\hline H II-1 & $2.40 \pm 0.07$ & $0.18 \pm 0.04$ & $0.25 \pm 0.06$ & $0.21 \pm 0.04$ & $0.19 \pm 0.05$ & - & $1.67 \pm 0.06$ \\
H II-2 & $2.27 \pm 0.09$ & $0.43 \pm 0.10$ & - & $0.27 \pm 0.11$ & - & - & $2.10 \pm 0.20$ \\
H II-3 & $2.40 \pm 0.68$ & - & - & $0.26 \pm 0.06$ & $0.26 \pm 0.08$ & - & $1.95 \pm 0.92$ \\
H II-4 & $3.28 \pm 0.06$ & $0.13 \pm 0.03$ & $0.42 \pm 0.02$ & $0.23 \pm 0.02$ & $0.19 \pm 0.01$ & $0.08 \pm 0.02$ & $1.48 \pm 0.03$ \\
H II-5 & $31.71 \pm 0.63$ & $1.00 \pm 0.09$ & $4.99 \pm 0.18$ & $1.91 \pm 0.08$ & $1.15 \pm 0.07$ & $0.75 \pm 0.16$ & $6.05 \pm 0.30$ \\
H II-6 & $2.89 \pm 0.06$ & $0.09 \pm 0.01$ & $0.45 \pm 0.02$ & $0.19 \pm 0.01$ & $0.12 \pm 0.01$ & $0.06 \pm 0.01$ & $1.37 \pm 0.03$ \\
H II-7 & $15.39 \pm 0.28$ & $0.58 \pm 0.08$ & $2.73 \pm 0.11$ & $0.98 \pm 0.05$ & $0.67 \pm 0.06$ & $4.00 \pm 0.41$ & $9.32 \pm 0.22$ \\
\hline
\end{tabular}

Table A.2. Metallicity obtained with different calibrations of the N2 and the O3N2 indicators.

\begin{tabular}{lcccccc}
\hline \hline Region & N2 (M13) & N2 (PP04) & O3N2 (M13) & O3N2 (PP04) & $R_{23}$ & $S_{23}$ \\
\hline H II-1 & $8.54 \pm 0.16$ & $8.68 \pm 0.18$ & $8.53 \pm 0.18$ & $8.73 \pm 0.14$ & $8.89 \pm 0.19$ & $8.36 \pm 0.20$ \\
H II-2 & $8.54 \pm 0.16$ & $8.69 \pm 0.18$ & $8.57 \pm 0.18$ & $8.79 \pm 0.14$ & $8.98 \pm 0.19$ & $8.18 \pm 0.21$ \\
H II-3 & $8.52 \pm 0.16$ & $8.64 \pm 0.18$ & $8.56 \pm 0.18$ & $8.78 \pm 0.14$ & $8.98 \pm 0.19$ & $8.20 \pm 0.20$ \\
H II-4 & $8.49 \pm 0.16$ & $8.58 \pm 0.18$ & $8.45 \pm 0.18$ & $8.60 \pm 0.14$ & $8.80 \pm 0.19$ & $8.33 \pm 0.20$ \\
H II-5 & $8.48 \pm 0.16$ & $8.56 \pm 0.18$ & $8.41 \pm 0.18$ & $8.55 \pm 0.14$ & $8.76 \pm 0.19$ & $8.46 \pm 0.20$ \\
H II-6 & $8.49 \pm 0.16$ & $8.58 \pm 0.18$ & $8.42 \pm 0.18$ & $8.56 \pm 0.14$ & $8.79 \pm 0.19$ & $8.52 \pm 0.20$ \\
H II-7 & $8.50 \pm 0.16$ & $8.60 \pm 0.18$ & $8.41 \pm 0.18$ & $8.55 \pm 0.14$ & $8.76 \pm 0.19$ & $8.51 \pm 0.20$ \\
\hline
\end{tabular}

Notes. For each indicator we list the value obtained using the Marino et al. (2013) method in Cols. 2 and 4 (M13), the Pettini \& Pagel (2004) method in Cols. 3 and 5 (PP04), the $R_{23}$ method (Pagel et al. 1979) for the upper limit in Col. 6 and the $S_{23}$ indicator (Pérez-Montero \& Díaz 2005) in Col. 7.

Table A.3. Elemental abundances obtained with the direct method (see text).

\begin{tabular}{|c|c|c|c|c|c|c|}
\hline Region & $12+\log (\mathrm{O} / \mathrm{H})$ & $12+\log (\mathrm{Ne} / \mathrm{H})$ & $12+\log (\mathrm{S} / \mathrm{H})$ & $12+\log (\mathrm{Ar} / \mathrm{H})$ & $12+\log (\mathrm{N} / \mathrm{H})$ & $\mathrm{He} / \mathrm{H}$ \\
\hline H II-1 & - & - & - & - & - & - \\
\hline H II-2 & - & - & - & - & - & - \\
\hline H II-3 & - & - & - & - & - & - \\
\hline H II-4 & $8.25 \pm 0.29$ & $7.49 \pm 0.74$ & - & - & - & - \\
\hline H II-5 & $8.10 \pm 0.14$ & $7.11 \pm 0.30$ & $6.91 \pm 0.09$ & $6.10 \pm 0.15$ & $7.61 \pm 0.18$ & $0.0809 \pm 0.0146$ \\
\hline H II-6 & $8.01 \pm 0.11$ & $6.99 \pm 0.22$ & $7.15 \pm 0.13$ & $6.32 \pm 0.18$ & - & $0.0849 \pm 0.0227$ \\
\hline H III-7 & $8.01 \pm 0.10$ & $7.05 \pm 0.24$ & $6.96 \pm 0.13$ & $6.11 \pm 0.24$ & - & $0.0706 \pm 0.0228$ \\
\hline
\end{tabular}

